\title{
Design of Shape Memory Alloy Sandwich Actuators: An Analytical and Numerical Modelling Approach
}

\author{
Luke Mizzi $^{1 *}$, Andrea Spaggiari ${ }^{1}$, Eugenio Dragoni ${ }^{1}$ \\ ${ }^{1}$ Department of Engineering Sciences and Methods, University of Modena and Reggio Emilia, \\ Reggio Emilia, Italy
}

*corresponding author email: luke.mizzi@unimore.it

\begin{abstract}
$\underline{\text { Abstract }}$
Shape memory alloy (SMA)-based actuator composites are characterised by a high force output which is activated by a temperature increase. In this work we exploit this property to design sandwich structures with SMA-matrix composite actuator skins capable of exhibiting a reversible, tailored flexural response. A theoretical model which predicts the resultant deflection and flexural moment produced as a result of selectively actuating one of the system skins was developed and confirmed using a multi-step Finite Element (FE) analysis which takes into account the fabrication pathway through which these systems may be manufactured. The model correlates the geometric parameters and material properties of the various components making up the system and provides a quantitative description of the role which each variable plays in determining the overall sandwich actuator performance. This is necessary for the future production and implementation of such systems in real-life applications.
\end{abstract}

Keywords: Shape Memory Alloys, Actuators, Composites, Analytical Modelling, Sandwich Structures

Nomenclature:

-

Force

Displacement

Applied pre-stretch force

$\begin{array}{ll}d_{i} & \text { Applied pre-stretch displacement } \\ k_{M} & \text { Stiffness of twinned martensitic st }\end{array}$

$k_{M} \quad$ Stiffness of twinned martensitic state of SMA component

$k_{T} \quad$ Stiffness of transition phase of SMA component

$k_{D M} \quad$ Stiffness of detwinned martensitic state of SMA component

$F_{T} \quad$ Force threshold required to undergo twinned martensitic to transition phase transformation

$d_{T} \quad$ Displacement threshold required to undergo twinned martensitic to transition phase transformation

$F_{D M} \quad$ Force threshold required to undergo transition phase to detwinned martensitic transformation

$d_{D M}$ Displacement threshold required to undergo transition phase to detwinned martensitic transformation

$k_{A} \quad$ Stiffness of initial linear austenitic phase of SMA component

$k_{\text {CB-Matrix }} \quad$ Axial stiffness of matrix counterbalance component

$k_{C B \text {-Sandwich }} \quad$ Cumulative stiffness of all sandwich structure components opposing SMA contraction 
$F_{E Q, C} \quad$ Cold state equilibrium force between SMA component and counterbalance

$d_{E Q, C} \quad$ Initial cold state equilibrium displacement between SMA component and counterbalance

$F_{E Q, H} \quad$ Hot state equilibrium force between SMA component and counterbalance of all sandwich components

sandwich components

$d_{E Q, H}^{\text {Ideal }} \quad$ Hot state equilibrium displacement between SMA component and matrix only (standalone $F_{\text {matrix }} \quad$ Axial force generated by matrix

$F_{\text {core }} \quad$ Axial component of flexural force generated by core

$F_{l s} \quad$ Axial component of flexural force generated by lower skin

$F_{A} \quad$ Morphing Force

$d_{A} \quad$ Actuation Stroke (Displacement)

$d_{A}^{\text {Ideal }} \quad$ Actuation Stroke (Displacement) of standalone actuator

$v \quad$ Poisson's Ratio

$E_{\text {matrix }} \quad$ Young's Modulus of matrix layer

$h_{\text {matrix }} \quad$ Length of matrix counterbalance component

E $\quad$ Young's modulus of sandwich core

$h_{\text {core }} \quad$ Thickness of sandwich core

$h_{S M A} \quad$ Thickness of SMA layer

$E_{A} \quad$ Young's modulus of initial linear austenitic state of SMA

$E_{M} \quad$ Young's modulus of twinned martensitic SMA

$E_{D M} \quad$ Young's modulus of detwinned martensitic SMA

$h \quad$ Hardening parameter

$R_{S} \quad$ Elastic limit

$e_{L} \quad$ Maximum transformation strain

$T_{0} \quad$ Reference conversion temperature

$\beta \quad$ Temperature scaling parameter

$\varepsilon_{i} \quad$ Applied pre-strain

$T_{f}$ 


\section{Introduction}

Shape memory alloys (SMAs) are materials that 'remember' a pre-set shape during loading, which is then recovered upon heating (1-5). Besides this shape memory effect, these smart materials also have the capability of exhibiting pseudoelastic behaviour at high temperatures. These remarkable thermomechanical and superelastic properties are related to the reversible nature of their phase transition between austenitic and martensitic states and make these systems ideal candidates for implementation in applications requiring materials which can tolerate relatively high strain deformations such as biomedical devices (6-9). Besides standalone systems, SMA have also been integrated in a number of composite systems in order to provide structural reinforcement $(10-15)$ as well as in sandwich structures (16-21) and laminates (22-24) to produce thermally-induced buckling and vibration dampening properties. In view of the unusual thermomechanical behaviour of these systems, a number of constitutive models and advanced numerical analysis techniques, including 1D, 2D and 3D methods, have also been developed specifically to investigate SMAs and related composite systems (25-32).

SMA-based composites have also found a wide range of applications in the field of actuation (33-36). SMAs are characterized by a high force to stroke ratio which may be activated by heating the system. However, this actuation effect may only be used once in stand-alone SMA system since upon cooling the system it does not return to its pre-stretched strain level. This shortcoming may be overcome by adding a secondary component to the system which provides a 'bias effect' that returns the system back to its original pre-strain shape, hence imparting reusability to the shape memory-induced actuation effect. This bias effect may be achieved through numerous methods which include the use of spring systems, fixed loads and even other SMA components (37-40). In SMA-based composite actuators made from SMA wires, this bias effect is imparted by the matrix in which the SMA component is embedded, which acts in a similar manner to an antagonistic elastic spring (41-44).

There are several methods through which one may design an SMA-based composite actuator. One such method is that proposed by Bettini et al. (42) where an array of SMA wires are fixed in a pre-stretched state and a matrix is formed by pouring the uncured resin around the clamped wires. Once the resin cures, the wires are released and the system equilibrates at a fixed displacement point, provided that there is a good level of adhesion between the two components of the composite system. The actuation stroke and reversibility of such an actuator depends on a number of factors with the most important being the volume fractions and material properties 
of the SMA and the matrix and the level of pre-strain applied to the SMA wires. It has been shown in a previous study by the same authors that through smart design of SMA-based composite actuators one may maximize the actuation potential of the system while retaining its reusability (45).

In this work, we present a design approach which may be used to implement these SMA-based composite actuators in sandwich structures with the aim of exploiting the axial actuation properties of these systems in order to obtain a reversible and tailored flexural response. By employing the actuators as upper and lower skins sandwiching a soft core, it is envisaged that by heating one of the faces of the sandwich structure, the actuator contracts axially resulting in a global flexural deformation of the entire sandwich system, which generates a resultant deflection that exceeds by far the corresponding axial displacement. In literature, SMA components in composite sandwich structures are primarily considered in terms of their ability to impart structural reinforcement, shape recovery functions and dampening properties rather than as a means of maximizing the actuation stroke potential of the sandwich system whilst ensuring reusability. Thus, in this work, we aim to focus mainly on the latter properties and, to this end, a theoretical model which quantitatively describes the extent of flexural deformation of the system based on the mechanical properties and volume fractions of the actuator skins and soft core was developed. The resultant analytical model predicts the maximum reversible deflection which may be achieved by the sandwich structure and was confirmed using Finite Element Analysis simulations. The design proposed in this work has the advantage that through its tri-material composition, the trade-off between maximum deflection and reversibility, which is one of the greatest limitations of a bi-material system, is removed. As a result of this, this SMA sandwich actuator is extremely versatile in terms of tailoring its actuation output and allows greater freedom of design to the user. Moreover, the model also takes into account the fabrication process used to produce the actuator, including the stepwise production and assembly of components and may be used as a direct design guide for these actuators.

\section{Finite Element Methodology}

A schematic of the sandwich structure investigated in this study and the main geometric parameters defining the system is presented in Figure 1. The system consists of a soft sandwich core and an upper and lower face, both made up of two layers of elastic matrix with a strip of SMA alloy embedded within. The overall thickness of the system, $H$, is the sum of the 
thicknesses of each layer, which are defined by $h_{\text {matrix }}, h_{S M A}$ and $h_{\text {core }}$, the heights of the matrix, SMA and core components making up the system, respectively, while the sandwich structure has a constant length, $L$, and out-of-plane thickness, $B$.

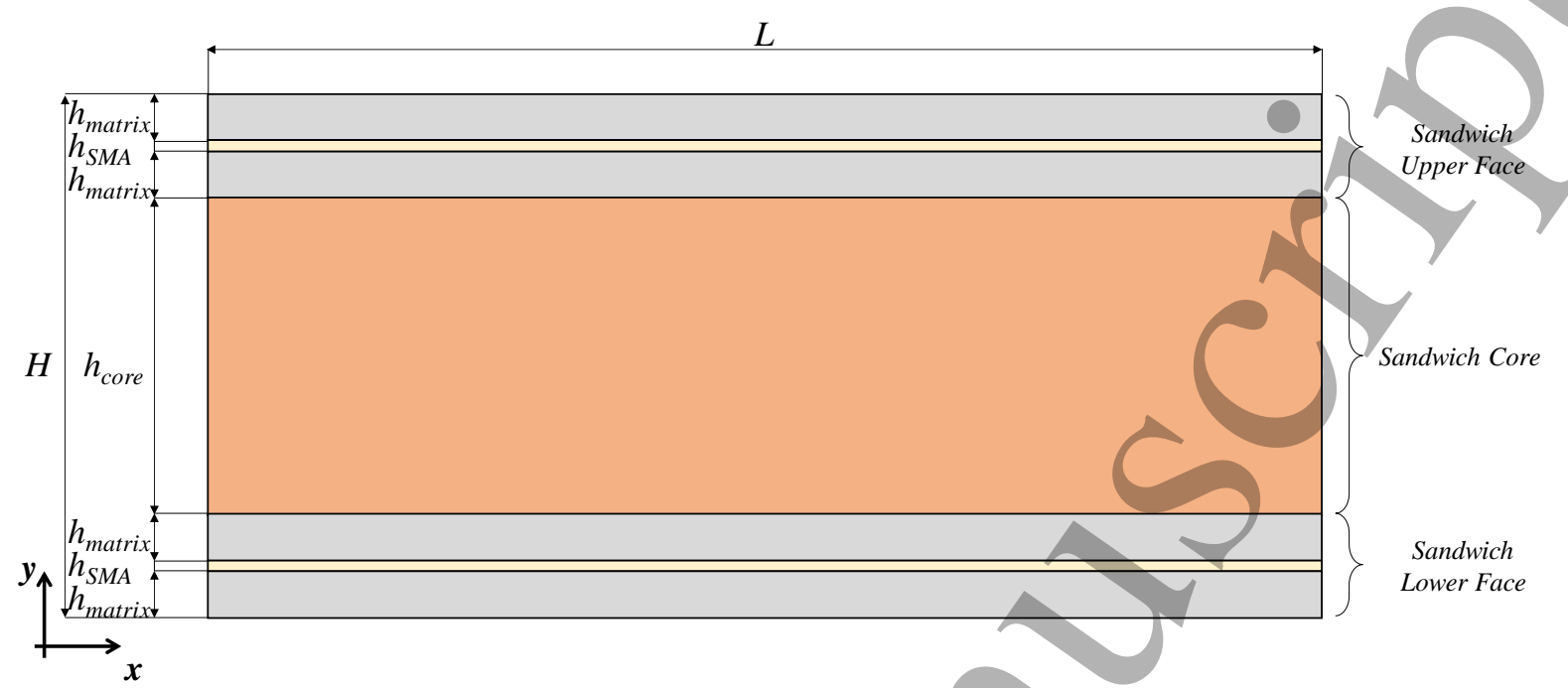

Figure 1: A 2D schematic of the SMA-based actuator sandwich structure showing the geometric parameters used to describe the system. The system's out-of-plane thickness is defined by $B$. The upper and lower sandwich faces are made up of two layers of elastic matrix with a small SMA strip embedded within.

Each of the three different layers within the system possess different material properties. The deformation of the matrix and core can each be described using a linear elastic model, defined by the Young's modulus, $E$, and Poisson's ratio, $v$. On the other hand, in the Finite Element simulations, the stress-strain behaviour of the SMA components was defined by the thermomechanical Souza-Auricchio Shape Memory model $(25,27)$, which takes into account the changes in stiffness associated with the martensitic and austenitic phases. This non-linear model defines the stress-strain behaviour of the SMA material through the following parameters: the Young's modulus of the austenitic and twinned martensitic state, $E_{M}$, the Young's modulus of the detwinned martensitic state, $E_{D M}$, the elastic limit, $R_{s}$, the maximum transformation strain, $e_{L}$, the hardening parameter, $h$, the reference conversion temperature, $T_{0}$, and the temperature scaling parameter, $\beta$. The Finite Element simulations were conducted using the ANSYS16 Multiphysics software and the sandwich structure was simulated as a system which is attached to an immovable surface from one side whilst the remaining sides remain free (see Figure 2). The simulations were conducted under plane-stress conditions, with large deflections allowed, and PLANE183 elements (8-noded quadratic plane-stress elements) were used to mesh the matrix and core, while PLANE223 (8-noded quadratic thermo-structural 
plane-stress elements) were used for the SMA strips. Following convergence tests, a minimum mesh thickness of $0.15 \mathrm{~mm}$ was used to simulate these systems.

\section{$\underline{\text { 2.1 Multi-step Finite Element Analysis }}$}

The simulation methodology employed to study these sandwich structures is based on the premise that the three separate components making up the system are manufactured individually and then joined together at the final production stage. This means that the actuator faces, which are made up of two components (i.e. SMA and matrix) that are under pre-stress, are first produced using a procedure similar to that described earlier and then later attached to the soft sandwich core, which is at rest. This is necessary in order to ensure that the sandwich actuator can be designed in a manner in which the thermally-induced actuation is completely reversible, with the matrix acting as the main bias component to the SMA and the core determining the extent of flexural deformation. In order to numerically analyse such a system using Finite Element Analysis, the following multi-step procedure, illustrated in Figure 2, was followed.

Initially, the sandwich system was constructed with an overall length of $l_{0}$ and set at a temperature of $T_{0}$, the reference martensitic temperature. In the first loadstep, which simulates the application of a pre-stress to the SMA strips, the matrix and core elements are switched off using the Element Death option available in ANSYS Multiphysics. Then, these elements are fixed as shown in Figure 2 in order to ensure that they do not undergo any extreme deformations while at the same time allowing the SMA strips to deform freely. These constraints were achieved through the implementation of displacement constraints in the $x$ direction applied on the leftmost edge of the entire system, while $y$-direction fixes were applied on the all the upper and lower edges of the matrix and core layers. In addition, the nodes on the rightmost edge of the system were coupled in order to allow them to deform concurrently in the $x$-direction. In order to induce a pre-stretch of the SMA strips, an axial tensile force, $F_{i}$, was applied on the nodes at the right edge of the SMA layers. Following the pre-stretching, which results in the simulated system having a total length of $l_{0}+d_{i}$, the next step was to simulate the formation of the matrix around the SMA and allow the system to equilibrate at a new displacement point, $d_{E Q, C 1}$. To this end, in Loadstep 2, the previously applied tensile forces and $y$-displacement fixes on the uppermost and lowermost edges of the system were removed and the matrix elements were switched on using Element Birth settings. Finally, in the third 
loadstep, the sandwich core was also switched on and the additional artificial constraints (couplings and fixes) on all elements were removed, leaving only the fixing constraints in the $x$-direction on the left edge of the system active as well as a single node on this edge which is fixed in both the $x$ - and $y$-directions in order to avoid rigid body motion. This loadstep simulates the behaviour of the sandwich structure upon heating the upper SMA strip to a higher final temperature, $T_{f}$, inducing an austenitic transformation which causes a flexural deformation.

\section{$\underline{2.2 \text { Geometric and Material Parameters }}$}

In the case studies investigated here, the SMA-matrix composite actuators making up the sandwich structure skins were designed according to the analytical expressions derived in (45). The theoretical model presented in that work provides a framework for the design of actuators with a tailored actuation stroke and complete reversibility for a relatively small number of cycles. In view of this, a matrix with the appropriate mechanical properties and volume fraction was chosen to complement the SMA material chosen; a Nitinol material whose thermomechanical properties were reported by Sittner et al. (46). The material properties of this SMA were replicated using the Souza-Auricchio model $(25,27)$ with the following parameters: $E_{A}=70 \mathrm{GPa}, h=0.5 \mathrm{GPa}, E_{D M}=70 \mathrm{GPa}, R=45 \mathrm{MPa}, e_{L}=0.03, T_{0}=253.15 \mathrm{~K}$ and $\beta=7.5 \mathrm{MPa} \mathrm{K}^{-1}$. This model describes the martensitic and austenitic stress-strain curves in a similar manner to the qualitative plot shown in Figure 3, and was chosen due to its suitability to model complex bi- and tri-dimensional shape memory behaviour. For the matrix, the Young's Modulus was set to $400 \mathrm{MPa}$ and the layer thicknesses of the SMA and matrix, $h_{S M A}$ and $h_{\text {matrix }}$, were set to $0.15 \mathrm{~mm}$ and $0.75 \mathrm{~mm}$ respectively. Since there are two layers of matrix, the final volume fraction of the SMA in the actuator skin consisted of $9.1 \%$. The initial length of the SMA strip, $l_{0}$, was set to $260 \mathrm{~mm}$ and a pre-stress equivalent to a pre-strain, $\varepsilon_{i}$ of 0.05 was applied to the system. Following the formation of the matrix and equilibration, this results in an initial sandwich structure length, $L$, of $267 \mathrm{~mm}\left(l_{0}+d_{E Q, C}\right)$ and a maximum actuation stroke of $6.37 \mathrm{~mm}$, which is completely reversible. While these parameters were all kept constant for all sandwich structures investigated in this work, a parametric study was conducted by changing the thickness, $h_{\text {core }}$, and Young's modulus of core, $E_{\text {core }}$, producing a wide variety of structures. More specifically, sandwich systems with cores possessing the following combination of geometric and material parameters were considered: $h_{\text {core }} \in 20 h_{\text {matrix }}$, 
$40 h_{\text {matrix }}, 60 h_{\text {matrix }}, 80 h_{\text {matrix }}$ and $E_{\text {core }} \in 10 \mathrm{MPa}, 20 \mathrm{MPa}, 40 \mathrm{MPa}$ and $80 \mathrm{MPa}$. Lastly, each of the three different materials used were assigned a Poisson's ratio, $v$, of 0.3 . 


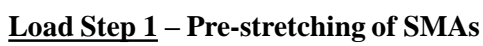

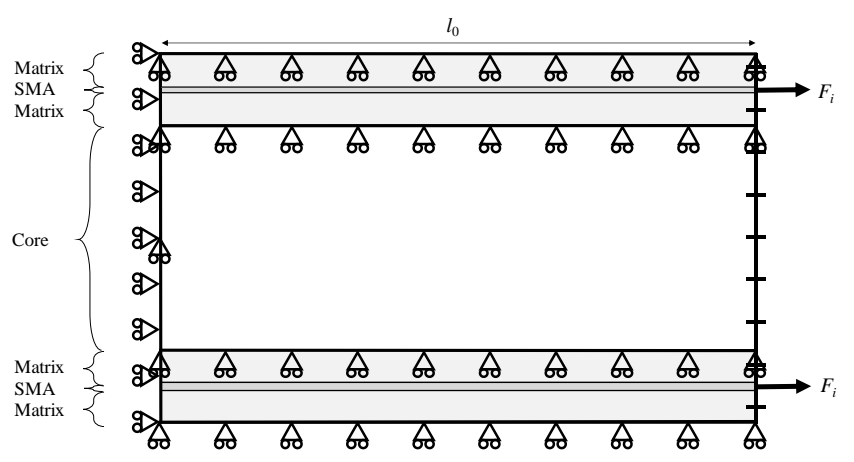

SMA Elements ON

Matrix and Core Elements OFF

Upper and Lower SMA Temperature $=T_{0}$

$\underline{\text { Load Step } 2}$ - Formation of Matrix

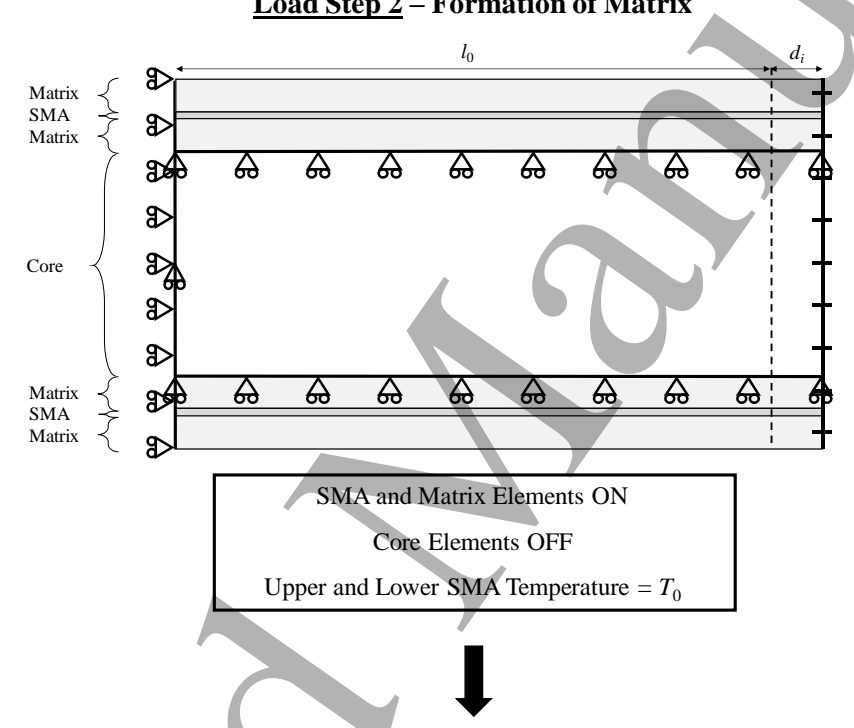

$\underline{\text { Load Step } 3}$ - Heating

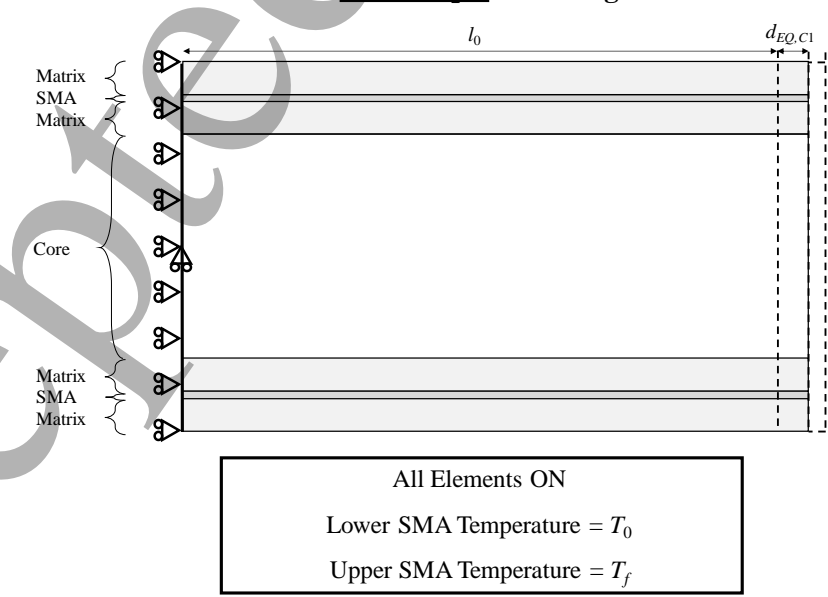

Figure 2: Diagram showing the three-step simulation procedure used to analyse the sandwich structures. The rollers represent fixes of the node displacements in the $x$ and $y$-directions, the crosses coupled nodes in the $x$-directions and the arrows in Loadstep 1 the application of forces in the $x$-direction on the surface nodes. 


\subsection{Post-Processing Analysis}

In order to measure the extent of flexural deformation of the sandwich structure, the deflection, $f$, and bending rotation, $\phi$, of the overall system were recorded during post-processing. The approximate overall deflection was measured as the $y$-displacement of topmost right edge node of the entire system, while the bending angle was calculated from the relative displacements of the rightmost edges of the upper and lower SMA layers using the following equation:

$$
\phi=\arctan \left(\frac{\left(x_{\text {lower }}+\Delta x_{\text {lower }}\right)-\left(x_{\text {upper }}+\Delta x_{\text {upper }}\right)}{\left(y_{\text {upper }}+\Delta y_{\text {upper }}\right)-\left(y_{\text {lower }}+\Delta y_{\text {lower }}\right)}\right)
$$

Eq. 1

where, $x_{\text {lower }}, x_{\text {upper }}, y_{\text {lower }}$ and $y_{\text {upper }}$ represent the initial $x$ and $y$ coordinates of the upper and lower edge nodes respectively and, the corresponding $\Delta x$ and $\Delta y$ terms, the displacements in the $x$ and $y$-directions of these nodes after deformation. In addition, for further analysis purposes, the changes in length of the upper and lower SMA strips were also recorded throughout every loadstep. In order to account for the curved deformation in Loadstep 3, the length was measured by summing up the distances between sequential nodes in the middle of the SMA layer using the following expression:

$$
l_{n}=\sum_{i=1}^{N-1} \sqrt{\left(\left(y_{i}+\Delta y_{i}\right)-\left(y_{i+1}+\Delta y_{i+1}\right)\right)^{2}+\left(\left(x_{i}+\Delta x_{i}\right)-\left(x_{i+1}+\Delta x_{i+1}\right)\right)^{2}}
$$

where, $N$ represents the total number of element nodes in a straight line, and $i$ and $i+1$ represent a pair of sequential nodes in the line.

Following the numerical simulations, the next step was to derive an analytical model capable of quantifying the extent of flexural deformation of these sandwich structures based on the simulation results obtained and observed deformation profiles.

\section{Analytical Model}

The theoretical model presented here is aimed at quantitively predicting the flexural deformation of the sandwich systems based on the geometric parameters and material properties of said structures. 


\section{$\underline{3.1 \text { Assumptions }}$}

The model is based on the assumption that:

a) a complete austenitic transformation of the SMA strip occurs upon heating,

b) there is perfect adhesion between each layer in the system,

c) the thickness of the skin layers $\left(2 h_{\text {matrix }}+h_{S M A}\right)$ is significantly smaller than that of the core $\left(h_{\text {core }}\right)$,

d) the core Young's modulus is lower than the matrix Young's modulus,

e) the sandwich system deforms as an eccentrically-loaded beam.

The reasoning behind the first assumption stems from the most common methodology used to heat SMA components in an actuator system; by passing a current through it. This typically results in a rise in temperature which by far exceeds that required to/achieve an austenitic transformation, even accounting for the fact that the application of a pre-stress raises the transformation temperature, and hence it may be safely assumed that if the systems studied here are designed to be activated in a similar manner incomplete phase transitions would not be an issue. Moreover, typical SMA wire or strips used for the design of heat-activated actuators are specifically chosen with a suitable temperature window for martensitic to austenitic transformation. The second, third and fourth assumptions are standard criteria for the design of functional sandwich structures. The layers of the sandwich structure must be well bonded to each other and there is no slippage between layers. Also, the faces of the sandwich structure must be thinner than the sandwich core which is typically made of a relatively soft material. Finally, the fifth assumption is a typical condition which is required to describe flexural deformation of sandwich and beam structures and is necessary if we are to analyse these systems using classic beam theory. This means that the length of the system, $L$, is significantly greater than the total thickness of the system, $H$ and thus a contraction of the upper SMA layer results primarily in bending deformation and that shear deformation is negligible. In fact, in the Finite Element simulations presented in the previous section, the most extreme cases considered had an $L / H$ rátio of at least 4 which is close to the lower limit in the theoretical analysis of beam structures (47). 


\subsection{Theoretical Model}

As mentioned previously in the methodology section, analytical expressions predicting the axial actuation stroke and reversibility of a standalone SMA-matrix composite actuator have already been reported (45). These expressions are based on the resolution of the individual axial force-displacement plots of the SMA and matrix components at low (martensitic) and high (austenitic) temperatures and in this work a similar approach will be followed as well to determine the actuation stroke of the SMA-composite actuator in the sandwich structure.

The martensitic force-displacement behaviour of the SMA component is described using a trilinear model, with three different stiffness constants for the twinned martensite, $k_{M}$, transition phase, $k_{T}$, and detwinned martensitic state, $k_{D M}$, as shown in Figure 3. The force-displacement relationship of the martensitic SMA changes from one stiffness constant to another upon reaching the transition force $\left(F_{T}\right)$ and the detwinned martensitic transformation force $\left(F_{D M}\right)$. The austenitic behaviour is described by a linear model with stiffness constant $k_{A}$ and the counterbalance, or matrix, stiffness is described by $k_{C B-M a t r i x}$. The mathematical relationships between these constants and the material and geometric parameters of the actuator skins are presented in (45) and the resultant equations for the plots shown in Figure 3 in terms of the latter set of parameters are shown in Eq. 3-8.

$$
\begin{aligned}
& F=\frac{B h_{S M A} E_{M}}{l_{0}} d \\
& F=\frac{B h_{S M A} E_{T}}{l_{0}} d+B h_{S M A}\left(\sigma_{T}-E_{T} \varepsilon_{T}\right) \\
& F=\frac{B h_{S M A} E_{D M}}{l_{0}} d+B h_{S M A}\left(\sigma_{D M}-E_{D M} \varepsilon_{D M}\right) \\
& F=\frac{B h_{S M A} E_{A}}{l_{0}} d \\
& F=-\frac{2 B h_{\text {matrix }} E_{\text {matrix }}}{l_{0}\left(\varepsilon_{i}+1\right)} d+\frac{2 B h_{\text {matrix }} E_{\text {matrix }} \varepsilon_{i}}{\varepsilon_{i}+1}
\end{aligned}
$$

Eq. 3

Eq. 6

where, $B$ represents the uniform out-of-plane thickness of the system, $E_{M}, E_{T}, E_{D M}$, and $E_{A}$ denote the twinned martensitic, transition, detwinned martensitic and austenitic phase Young's moduli respectively, $\sigma_{T}, \sigma_{D M}, \varepsilon_{T}$ and $\varepsilon_{D M}$ the phase transformation stresses and strains between twinned martensitic and transition phases and transition and detwinned martensitic phases, 
respectively. The level of pre-strain applied to the system is denoted by $\varepsilon_{i}$ and the Young's modulus of the matrix by $E_{\text {matrix. }}$ Note that Eq. 7 has been adapted from the form presented in (45) since in the sandwich structures considered here the geometric parameter $h_{\text {matrix }}$ represents half the volume fraction of the matrix in the entire actuator rather than all of it.

In a standalone actuator, the matrix provides the sole opposing force to the SMA component at all stages. Thus, the first cold $\left(d_{E Q, C 1}, F_{E Q, C 1}\right)$ and heated $\left(d_{E Q, H}, F_{E Q, H}\right)$ equilibration points are defined by solving Eq. 5 and 7 and Eq. 6 and 7 respectively, with the actuation stroke, $d_{A}$, being the difference between $d_{E Q, C 1}$ and $d_{E Q, H}$. However, in the case of the sandwich structures considered here, the sandwich core along with the other actuator face are attached to the system after the first equilibration point. This means that while $\left(d_{E Q, C 1}, F_{E Q, C 1}\right)$ is determined only by the level of applied pre-strain, geometric parameters and material properties of the SMA and matrix, the hot equilibration point $\left(d_{E Q, H}, F_{E Q, H}\right)$ is dependent on the sum of the counterbalance axial forces exerted by both the matrix and the rest of the sandwich structure. This also means that the reversibility potential of the actuator is entirely independent of the overall sandwich geometry since it depends only on the position of the first cold equilibration point $\left(d_{E Q, C 1}\right.$, $\left.F_{E Q, C 1}\right)$. If $F_{E Q, C 1}$ is greater than $F_{D M}$, then the actuation stroke can be completely recovered after re-cooling the system i.e. $\left(d_{E Q, C 1}, F_{E Q, C 1}\right)=\left(d_{E Q, C 2}, F_{E Q, C 2}\right)$. Hence, assuming that the actuator skin used adheres to this condition, the cold equilibration point of the system $\left(d_{E Q, C}\right.$, $\left.F_{E Q, C}\right)$ may be found by solving Eq. 5 and 7, which results in:

$$
\begin{aligned}
F_{E Q, C} & =\frac{2 B h_{\text {matrix }} E_{\text {matrix }} h_{S M A}\left(E_{D M} \varepsilon_{i}+\sigma_{D M}-E_{D M} \varepsilon_{D M}\right)}{E_{D M} \varepsilon_{i} h_{S M A}+E_{D M} h_{S M A}+2 E_{\text {matrix }} h_{\text {matrix }}} \\
d_{E Q, C} & =\frac{l_{0}\left(E_{D M} \varepsilon_{D M} \varepsilon_{i} h_{S M A}+E_{D M} \varepsilon_{D M} h_{S M A}+2 E_{\text {matrix }} \varepsilon_{i} h_{\text {matrix }}-\varepsilon_{i} h_{S M A} \sigma_{D M}-h_{S M A} \sigma_{D M}\right)}{E_{D M} \varepsilon_{i} h_{S M A}+E_{D M} h_{S M A}+2 E_{\text {matrix }} h_{\text {matrix }}}
\end{aligned}
$$




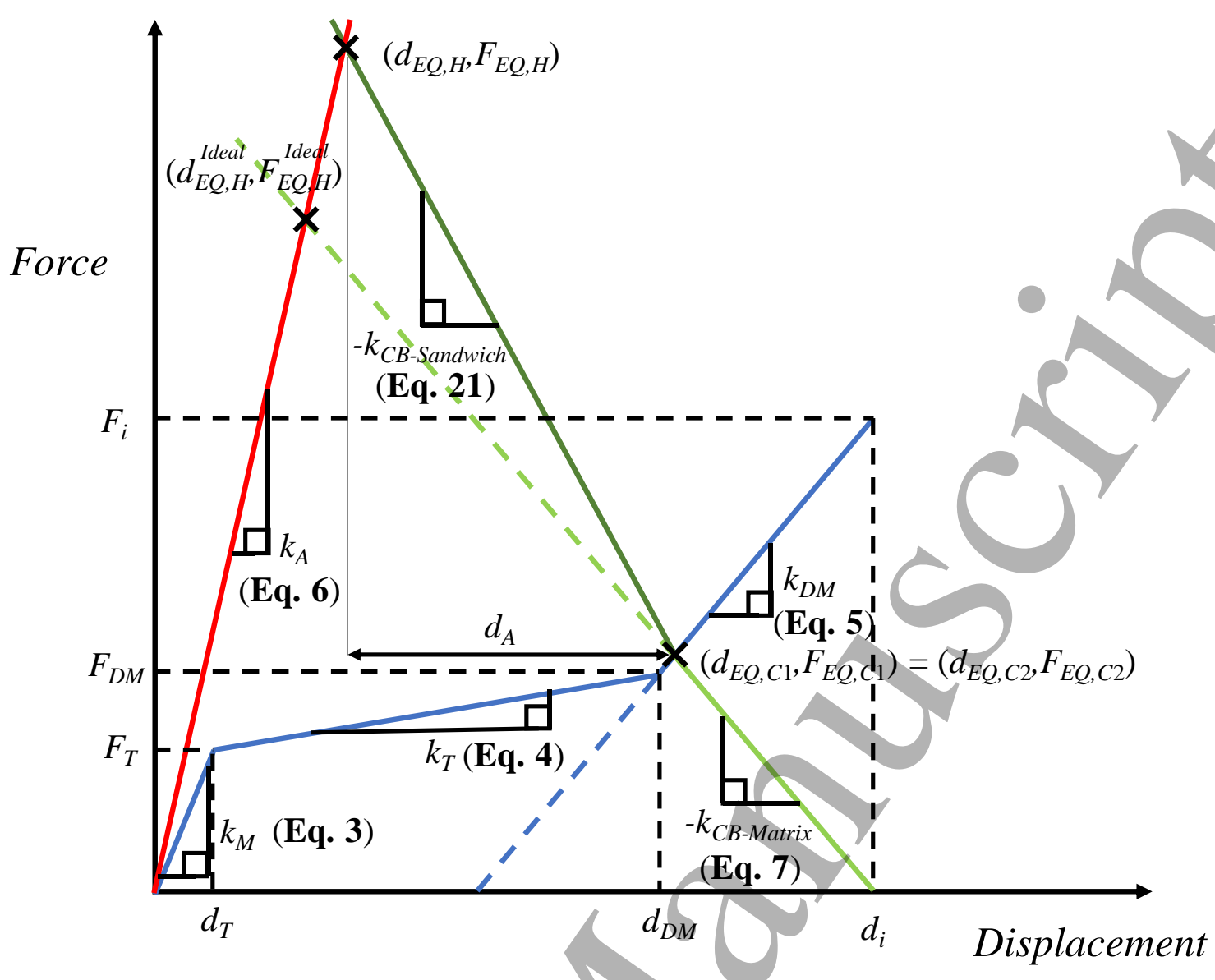

Figure 3: Qualitative plot showing the axial force-displacement relationships governing the SMA-matrix composite actuator skin of the sandwich system.

Eq. 9 is required to define the initial length of the overall sandwich system., $L$, which is given by:

$$
L=l_{0}+d_{E Q, C}
$$

Eq. 10

The equations up to this point describe the formation of the sandwich structure. The displacements and forces calculated thus far all act in the axial direction parallel to the layering of the system, which is aligned with the $x$-direction in a global cartesian system. Following the application of heat to the upper SMA strip, the system behaves as if it is loaded with an eccentric axial force aligned with upper wire. Therefore, the system undergoes a pure bending moment and the axial force and the shape of the sandwich can be described considering a cylindrical coordinate system with radius, $R$, which is dependent on the bending angle, $\phi$ (see Figure 4). 


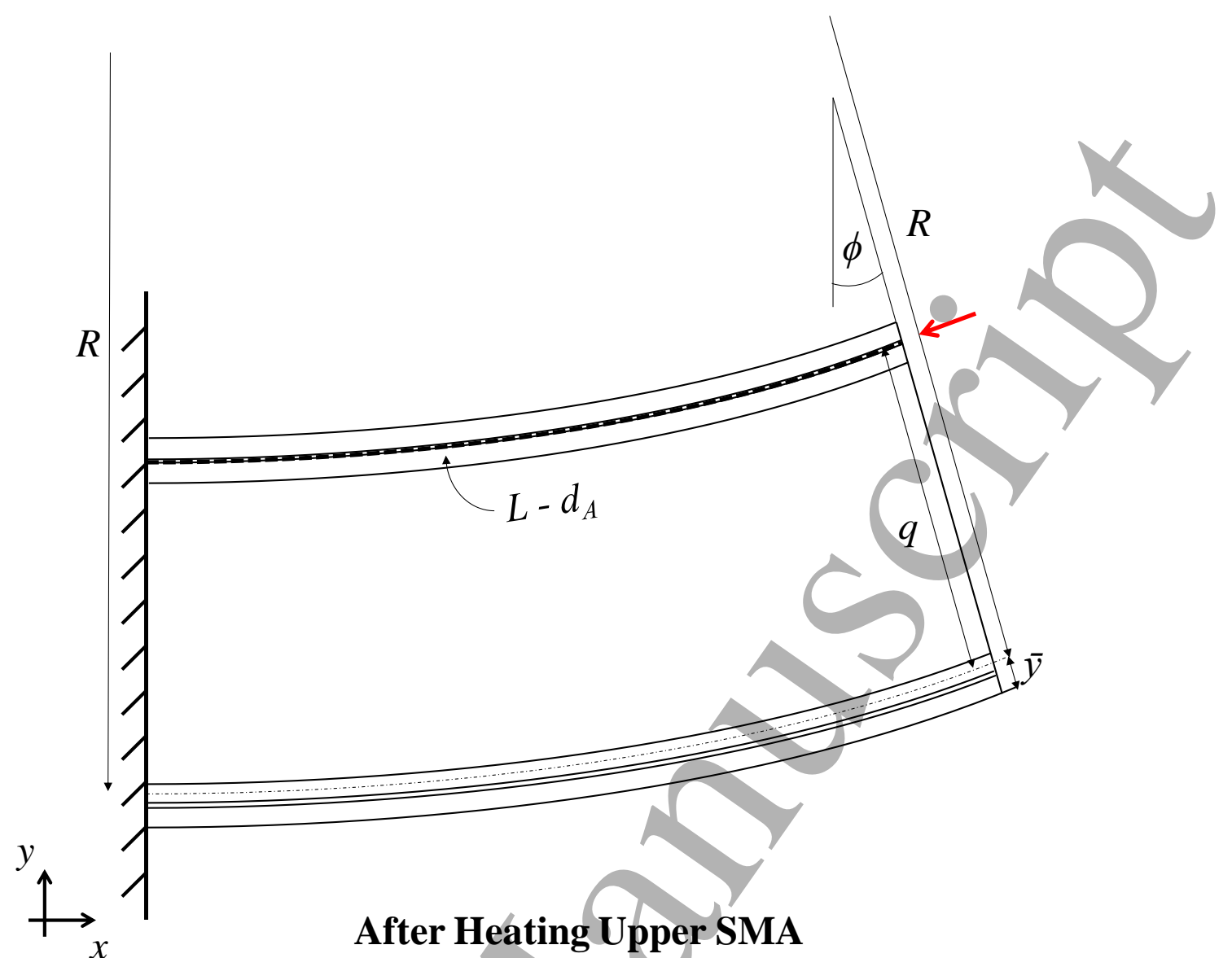

Figure 4: A schematic of the sandwich structure after heating of the upper SMA strip. The red arrow indicates the direction along which the force shown in the plot in Figure 5 is aligned, i.e. the cylindrical axis with radius $R$. The actuation displacement, $d_{A}$, is also measured along this direction.

The loading method applied to the sandwich structure to induce deformation, i.e. the application of heat to one SMA layer only, is inherently asymmetric. Besides being similar to the application of an eccentric load to the sandwich structure, this method also affects the position of the centroid and neutral axis of deformation within the system. This is due to the fact that while the SMA layer in the upper skin induces the flexural deformation of the system, the entire lower skin of the sandwich structure opposes it. This generates an asymmetry in the stiffness distribution of the system despite the geometric symmetry along the $x z$-plane which the system possesses, resulting in a displacement of the centroid from the centre of the system. The position of centroid relative to the base of the sandwich structure, $G$, may be found as follows (48): 


$$
G=\frac{\left(2 h_{\text {matrix }}+h_{S M A}\right) \frac{E_{\text {matrix }}}{E_{\text {core }}}\left(\frac{3}{2} h_{S M A}+3 h_{\text {matrix }}+h_{\text {core }}\right)+h_{\text {core }}\left(h_{S M A}+2 h_{\text {matrix }}+\frac{h_{\text {core }}}{2}\right)+\left(2 h_{\text {matrix }}+h_{S M A}\right) \frac{E_{\text {Cold }}^{*}}{E_{\text {core }}}\left(\frac{h_{S M A}}{2}+h_{\text {matrix }}\right)}{\left(2 h_{\text {matrix }}+h_{S M A}\right) \frac{E_{\text {matrix }}}{E_{\text {core }}}+h_{\text {core }}+\left(2 h_{\text {matrix }}+h_{S M A}\right) \frac{E_{\text {Cold }}^{*}}{E_{\text {core }}}}
$$

Eq. 11

where, $E_{\text {Cold }}^{*}$ represents the effective Young's modulus of the lower actuator skin in the $x$ direction which is made up of martensitic SMA and matrix layers. This parameter may be calculated as follows:

$$
E_{\text {Cold }}^{*}=\left(E_{D M} \frac{h_{S M A}}{h_{S M A}+2 h_{\text {matrix }}}+\frac{E_{\text {matrix }}\left(1-\frac{h_{S M A}}{h_{S M A}+2 h_{\text {matrix }}}\right)}{1+\varepsilon_{i}}\right)\left(\frac{d_{E Q, C}}{l_{0}}+1\right)
$$

Eq. 12

Since the lower skin is typically much stiffer than upper skin matrix layers and the sandwich core, the centroid will normally shift to a region inside or close to the lower skin.

When the upper SMA layer is heated, it contracts axially. Since the matrix layers surrounding the SMA are in its immediate vicinity and very thin, the force generated by these layers opposing the axial contraction of the SMA may also be calculated axially using Eq. 7. However, the same cannot be said for the lowerskin and the sandwich core and therefore the opposing axial forces in the direction and plane of the upper SMA generated by these layers must be calculated using beam theory. The flexural moment, $M_{f}$, induced by the axial actuation force of the SMA may be quantified as beam loading under eccentric conditions as follows:

$$
M_{f}=F q
$$

Eq. 13

where, $q$, is the distance between the centre of the upper SMA layer and the neutral axis of the system. The position of the neutral axis of the system is displaced from the centroid, $G$, due to the eccentricity, $e$, which is defined as the distance between the upper SMA layer and the centroid:

The displacement of the neutral axis from the centroid due to the eccentricity, $y_{n}$, can be found by: 


$$
y_{n}=\frac{i^{2}}{e}
$$

with $i^{2}$ being the square of the gyration radius of the sandwich structure, which is in turn calculated from the effective second moment of inertia of the sandwich structure, $I^{*}$, and the effective cross-sectional area, $A^{*}$ :

$$
i^{2}=\frac{I^{*}}{A^{*}}
$$

Eq.16

and the parameters $I^{*}$ and $A^{*}$ are defined as:

$$
\begin{aligned}
& I^{*}=B\left[\begin{array}{l}
\left(\frac{\left(2 h_{\text {matrix }}+h_{S M A}\right)^{3}}{12}+\left(2 h_{\text {matrix }}+h_{S M A}\right) \frac{E_{\text {matrix }}}{E_{\text {core }}}\left(3 h_{\text {matrix }}+\frac{3}{2} h_{\text {SMA }}+h_{\text {core }}-G\right)^{2}\right) \\
+\left(\frac{\left(h_{\text {core }}\right)^{3}}{12}+h_{\text {core }}\left(2 h_{\text {matrix }}+h_{S M A}+\frac{h_{\text {core }}}{2}-G\right)^{2}\right) \\
+\left(\frac{\left(2 h_{\text {matrix }}+h_{\text {SMA }}\right)^{3}}{12}+\left(2 h_{\text {matrix }}+h_{S M A}\right) \frac{E_{\text {cold }}^{*}}{E_{\text {core }}}\left(h_{\text {matrix }}+\frac{h_{S M A}}{2}-G\right)^{2}\right)
\end{array}\right] \\
& A^{*}=B\left[\begin{array}{l}
\left.\left(2 h_{\text {matrix }}+h_{S M A}\right) \frac{E_{\text {matrix }}}{E_{\text {core }}}+h_{\text {core }}+\left(2 h_{\text {matrix }}+h_{\text {SMA }}\right) \frac{E_{\text {cold }}^{*}}{E_{\text {core }}}\right]
\end{array}\right.
\end{aligned}
$$

Eq. 17

Eq. 18

Using these parameters, the position of the neutral axis with respect to the lower edge of the sandwich structure, $\bar{y}$, may be found by:

$$
\bar{y}=G-y_{n}
$$

Eq. 19

and $q$ is defined as:

$$
q=e+y_{n}
$$

Eq. 20

The flexural moment of the sandwich structure may be related to the flexural radius, $R$ (which is relative to the neutral axis), as follows:

$$
M_{f}=\frac{E I}{R}
$$


where $E$ is the Young's modulus and $I$ is the second moment of inertia of the beam section being considered. The radius, $R$, is related to the axial contraction of the SMA strip, $d$, through Eq. 16:

$$
R=\frac{L q}{d}
$$

By expanding Eq. 13 and 22, the axial force, $F$, and displacement, $d$, generated by the flexural deformation of any of the system layers at a distance $e$ away from the neutral axis may be found by:

$$
F=\frac{E I}{L q^{2}} d
$$

Eq. 23

In order to calculate the flexural stiffness of the sandwich core and the lower skin, the respective moments of inertia of these two components must first be calculated as follows:

$$
\begin{aligned}
& I_{\text {core }}=B\left(\frac{\left(h_{\text {core }}+h_{S M A}+2 h_{\text {matrix }}-\bar{y}\right)^{3}}{3}-\frac{\left.\left(h_{S M A}+2 h_{\text {matrix }}-\bar{y}\right)^{3}\right)}{3}\right) \\
& I_{l s}=B\left(\frac{\left(h_{S M A}+2 h_{\text {matrix }}-\bar{y}\right)^{3}}{3}-\frac{(-\bar{y})^{3}}{3}\right)
\end{aligned}
$$

where $B$ is the $z$-thickness of the sandwich structure. Finally, since the force-displacement plots generated by these components both originate from the first equilibration point $\left(d_{E Q, C}, F_{E Q, C}\right)$ shown in Figure 3, the opposition forces generated as the upper SMA strip contracts may be found by:

$$
\begin{aligned}
& F_{\text {core }}=\frac{E_{\text {core }} I_{\text {core }}}{L q^{2}}\left(d_{E Q, C}-d\right) \\
& F_{l s}=\frac{E_{c o l d}^{*} I_{l s}}{L q^{2}}\left(d_{E Q, C}-d\right)
\end{aligned}
$$

And the total counter balance force may be found by adding Eq. 7 to Eq. 26 and 27:

$$
F=F_{\text {matrix }}+F_{\text {core }}+F_{l s}
$$

Finally, the hot equilibration point $\left(d_{E Q, H}, F_{E Q, H}\right)$ may be found by solving Eq. 6 and 28: 


$$
\begin{gathered}
F_{E Q, H}=\frac{\frac{d_{E Q, C}}{L q^{2}}\left(E_{\text {core }} I_{\text {core }}+E_{\text {cold }}^{*} I_{l s}\right)+\frac{2 B h_{\text {matrix }} E_{\text {matrix }} \varepsilon_{i}}{\varepsilon_{i}+1}}{1+\frac{l_{0}}{B h_{S M A} E_{A}}\left(\frac{1}{L q^{2}}\left(E_{\text {core }} I_{\text {core }}+E_{\text {cold }}^{*} I_{l s}\right)+\frac{2 B h_{\text {matrix }} E_{\text {matrix }}}{l_{0}\left(\varepsilon_{i}+1\right)}\right)} \\
d_{E Q, H}=\frac{\frac{d_{E Q, C}}{L q^{2}}\left(E_{\text {core }} I_{\text {core }}+E_{\text {cold }}^{*} I_{l s}\right)+\frac{2 B h_{\text {matrix }} E_{\text {matrix }} \varepsilon_{i}}{\varepsilon_{i}+1}}{\frac{B h_{S M A} E_{A}}{l_{0}}+\left(\frac{1}{L q^{2}}\left(E_{\text {core }} I_{\text {core }}+E_{\text {cold }}^{*} I_{l s}\right)+\frac{2 B h_{\text {matrix }} E_{\text {matrix }}}{l_{0}\left(\varepsilon_{i}+1\right)}\right)}
\end{gathered}
$$

Eq. 30

Once the hot equilibrium point has been found, the axial actuation stroke of the heated SMA layer, $d_{A}$, can be found by:

$$
d_{A}=d_{E Q, C}-d_{E Q, H}
$$

Eq. 31

The actuation stroke can then be used to calculate the bending angle, $\phi$, and deflection, $f$, as follows:

$$
\begin{aligned}
& \phi=\frac{d_{A}}{q} \\
& f=2\left(\frac{L q}{d_{A}}-H+\bar{y}\right) \sin ^{2}\left(\frac{d_{A}}{2 q}\right)
\end{aligned}
$$

where, $H$ represents the total thickness of the sandwich system.

\section{Results and Discussion}

A diagram showing the deformation of one of the sandwich structures studied here before and after Loadstep 3 of the FE simulation is shown in Figure 5. A comparison between the FE results and the theoretical predications is also presented in Figure 6, where the effect of changes in the parameters $E_{\text {core }}$ and $h_{\text {core }}$ on the deflection, $f$, for a specific set of geometrical parameters, are presented. The analytical model and Finite Element simulations show extremely good agreement, indicating that the theoretical model covers and accurately predicts the geometric and material nonlinearities of the system. It is evident that the largest deflections are obtained from the systems with the lowest $h_{\text {core }}$ value. This was not surprising since for a given $d_{\AA}$ value, the axial actuation stroke of the SMA-matrix actuator, the greater the $d_{A} / H$ 
ratio, the larger the extent of bending rotation, $\phi$, and hence flexural deflection, $f$. Moreover, it was also observed that in most cases the Young's modulus of the core had minimal effect on the extent of flexural deformation, except in systems possessing an extremely rigid core, in which case, a small reduction was observed.

Figure 5: A scale image of a Finite Element simulation on a system with $E_{\text {core }}=20 \mathrm{MPa}$ and $\boldsymbol{h}_{\text {core }}=20 \boldsymbol{h}_{m}$ before (dotted line) and after heating the uppermost SMA layer.

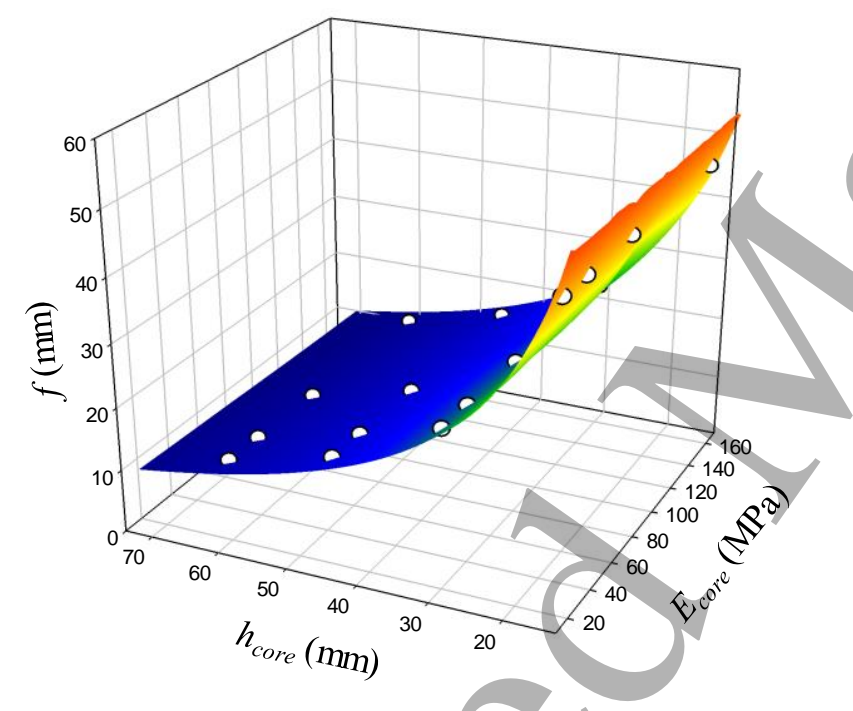

Constant Geometric Parameters

Figure 6: Plot showing the changes in actuation deflection, $f$, obtained upon changing the parameters $\boldsymbol{h}_{\text {core }}$ and $E_{\text {core }}$ whilst keeping all other parameters constant as predicted by the analytical model (surface) (Eq. 33) and FE simulations (points). The deflection observed for these actuators is predicted to be fully reversible.

This indicates that the reaction force imparted by the core against the axial contraction, $F_{\text {core }}$, of the heated SMA strip is minimal in most cases. This is the most desirable outcome, since in an ideal situation, the only role of the core is to act as a geometric parameter which transforms the axial contraction of the skin into an overall flexural deformation of the sandwich structure without reducing the actuation stroke of the skin layer. This means that the total counterbalance stiffness, $k_{C B \text {-Sandwich }}$, must be almost equal to $k_{C B \text {-Matrix }}$ (see Figure 3 ), i.e. $F_{\text {matrix }} \approx F_{\text {matrix }}+F_{\text {core }}$ 
$+F_{l s}$. In order to minimize the value of $F_{\text {core }}$, the flexural stiffness of the core must be very low. Since the $d_{A} / H$ ratio determines the extent of flexure, if $h_{\text {core }}$ is kept constant, then $E_{\text {core }}$ must be accordingly low for this to occur. However, it must be noted that the influence of a fixed $E_{\text {core }}$ on the overall flexural stiffness of the core increases as $h_{\text {core }}$ increases since the second moment of inertia increases by the power of three with increasing core thickness (see Eq. 18). This is amply demonstrated in Figure 7, where while the systems with an $E_{\text {core }}$ value of 10 MPa show no significant effect on the axial actuation stroke of the SMA-matrix skin, those with an $E_{\text {core }}$ value of $80 \mathrm{MPa}$ show a slight decrease. The reason why the stiffness imparted by the sandwich core cannot be neglected in the cases studied here as in normal sandwich structures subjected to external loads is due to the fact that the neutral axis of the system is displaced from the centre towards the unheated skin of the sandwich structure. However, on the other hand, the flexural stiffness of the lower skin is very small, since it is relatively thin and extremely close to the neutral axis of rotation in almost all cases. In fact, almost no change in the length of the unheated SMA strip was observed from the FE results.

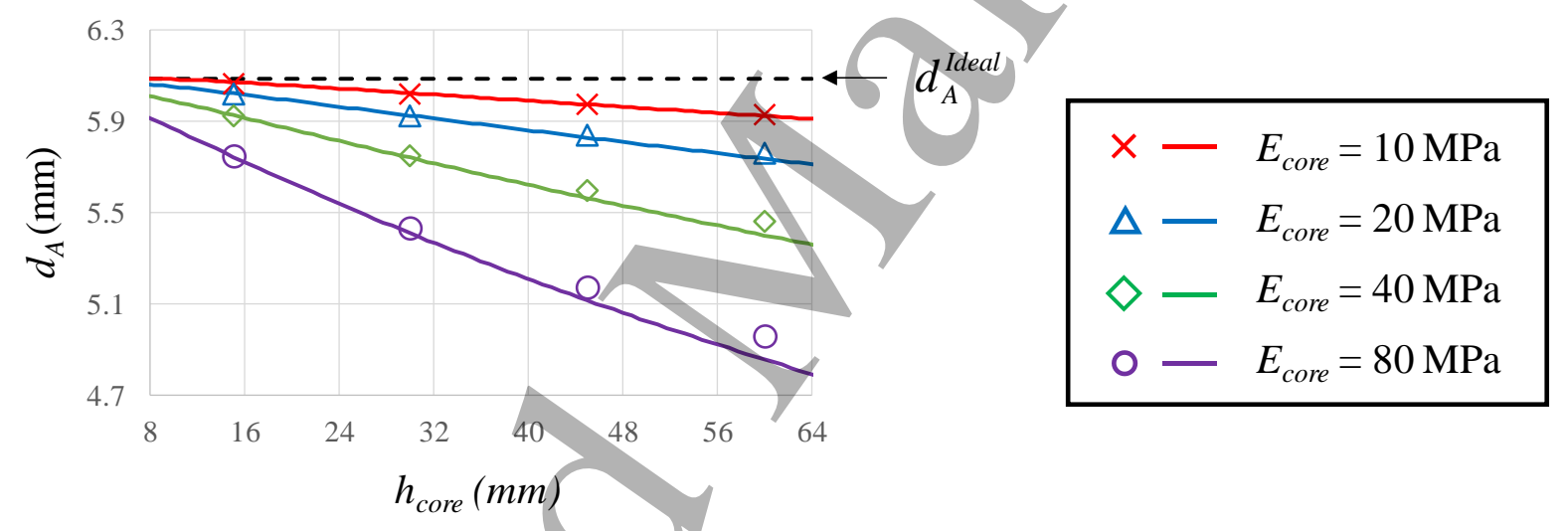

Figure 7: Plot showing how the axial actuation stroke of the heated SMA changes with changing $\boldsymbol{h}_{\text {core }}$ for various sandwich structures as predicted by the analytical model (straight lines) and FE simulations (symbols).

Therefore, if one wishes to quantify the reduction of actuation stroke, $d_{A}$, as a result of the core stiffness, then one must first solve Eq. 6 and 7 in order to get the ideal hot equilibrium point, $d_{E Q, H}^{\text {Ideal }}$, i.e. the equilibrium point of a standalone SMA-matrix actuator:

$$
d_{E Q, H}^{\text {Ideal }}=\frac{2\left(\frac{h_{\text {matrix }} E_{\text {matrix }} \varepsilon_{i}}{\varepsilon_{i}+1}\right)}{\left(\frac{h_{S M A} E_{A}}{l_{0}}+2\left(\frac{h_{\text {matrix }} E_{\text {matrix }}}{l_{0}\left(\varepsilon_{i}+1\right)}\right)\right)}
$$


which may then be used to calculate the ideal actuation, $d_{A}^{\text {Ideal }}$ :

$$
d_{A}^{\text {Ideal }}=d_{E Q, C}-d_{E Q, H}^{\text {Ideal }}
$$

Finally, the actuation efficacy factor, $A_{\text {eff }}$, may be found by comparison with Eq. 31:

$$
A_{\text {eff }}=1-\left(\frac{d_{A}^{\text {Ideal }}-d_{A}}{d_{A}^{\text {Ideal }}}\right)
$$

Eq. 36

If $A_{\text {eff }} \rightarrow 1$, then the actuation output of the skin is at its maximal level and the sandwich core has solely a geometric effect on the deflection of the overall system. When constructing such sandwich systems, one should optimise the necessary geometric parameters and material properties of the various components making up the system bearing this factor in mind in order to obtain the maximal actuation efficacy.

At this point, it is important to highlight the critical role that the core plays on the overall deformation of the sandwich structure. As stated previously, if acting solely in a geometric capacity, the presence of a thick core actually decreases the deflection of the sandwich structure produced upon actuation. However, conversely, the flexural moment generated by the entire sandwich system increases upon increasing core thickness. This relationship between flexural moment and deflection, quantified in Figure 8, is similar to that observed in many actuator systems, where a large stroke is typically accompanied by a small force and vice-versa. Moreover, the sandwich core also acts as a barrier against shear deformation of the system upon actuation of the SMA layer. 


\section{Constant $E_{\text {core }}=20 \mathrm{MPa}$}
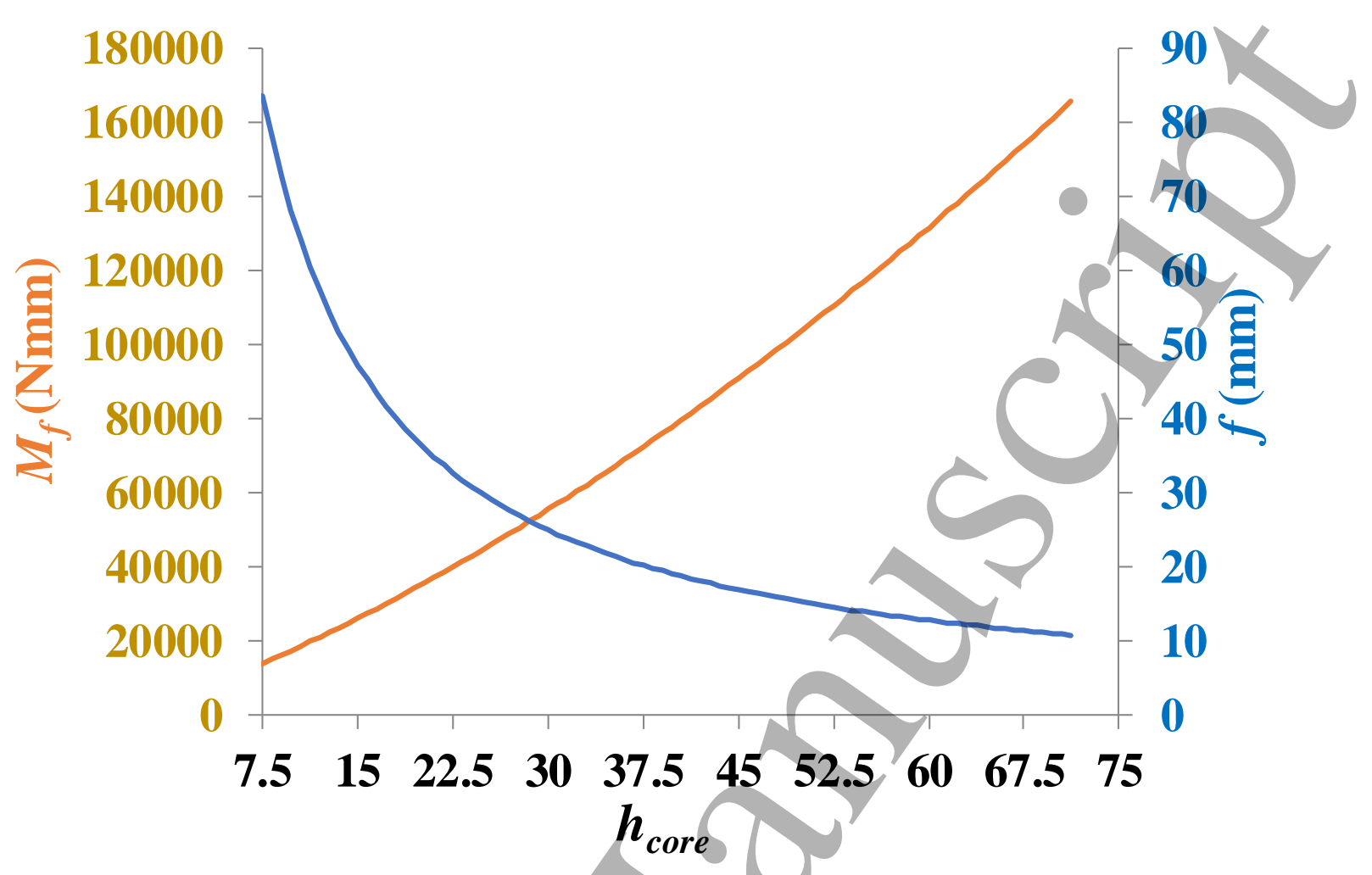

Figure 8: Plot showing the inversely proportional relationship between flexural moment, $M_{f}$, and deflection, $f$, upon altering the core thickness, $h_{\text {core, }}$, and leaving all other parameters constant (with $B$ set to $100 \mathrm{~mm}$ ), as predicted by the theoretical model.

The plots shown in Figures $\mathbf{7}$ and $\mathbf{8}$ also highlight the very clear advantage which a sandwich actuator has over a standalone axial SMA actuator with respect to the overall actuation stroke output of the system. While the maximum actuation stroke generated by the standalone actuator for the examples considered here is $6.37 \mathrm{~mm}$, the resultant deflection of the corresponding sandwich structures incorporating the same actuator as a component of the system can vary from $c a .11 \mathrm{~mm}$ to $90 \mathrm{~mm}$, depending on the type of sandwich core used. These higher values, although coming at a cost of the overall actuation moment and force of the system, considerably enhance the versatility and applicability of SMA-based sandwich actuators, particularly for use in applications requiring proportionate stroke to force actuation ratios. It is also worth highlighting that his increased actuation stroke does not come at a cost of reusability. In fact, for systems designed with an $A_{\text {eff }}$ factor of almost 1 , the reversibility of the system upon cooling is unaffected by the maximum permissible stroke, which is primarily defined by the core thickness. This is not the case for a bi-material actuator, where the matrix acts both as a bias 
system and a sole determinant of the maximum actuation stroke, hence resulting in a trade-off between these two factors which limits the versatility of the actuator.

Before concluding, it is imperative to mention that the analytical model and Finite Element simulations presented here are meant primarily to act as a pre-design guide for the fabrication of SMA actuator-based sandwich structures. It is well known that the stress-strain behaviour of SMAs is affected by long-term repeated use (49-51) and that fatigue factors need to be taken into consideration if the model presented here is to be used to predict the behaviour of actuator systems operating under these conditions. Therefore, the model is mainly expected to accurately predict the behaviour of these systems for short-term repeated use, particularly if materials with a short fatigue lifetime are used. Moreover, the choice of materials used to produce these sandwich structures is also essential to obtaining the desired functionality. Finding a set of materials which when joined together respect all of the conditions listed in the model section can be a challenge, in particular for the condition of ensuring perfect adhesion between all layers. Other factors such as manufacturing conditions including curing process of matrix such as temperature changes and shrinkage could also result in slight deviations from the theoretical predictions of this model. A possible method to circumvent this problem and ensure that the matrix acts as a bias mechanism for the SMA component in these cases and/or in the event of imperfect adhesion between layers could be to mechanically block the ends of the SMA layers using a rigid element that allows uniform compression of the sandwich skins. The analytical model presented here combines the effect of the geometric parameters with the material properties of the various components making up the system in order to obtain a desired mechanical response and thus is expected to provide the necessary design flexibility required to overcome such considerations through optimization of the geometric variables based on the material properties of the suitably chosen materials. Furthermore, it should be noted that while the model presented here is based on 3D system with a constant out-of-plane cross-section for the sake of clarity, the equations provided may easily be slightly tweaked to describe a system where the SMA component is in the form of a wire rather than a strip of material, as is the case in most SMA-based composite actuators. This may be achieved by taking into account the relative volume fractions of the SMA and matrix components based on their cross-sectional areas rather than thickness, whilst retaining roughly the same overall skin thickness. The next step in this work is to design a prototype sandwich actuator based on the model proposed here and use it to experimentally validate the conclusions of this numerical and analytical study. 


\section{Conclusions}

In this work, analytical expressions relating to the design of SMA actuator-based sandwich structures with a reversible tailored flexural response has been presented. The theoretical model describes the effect of the various geometric parameters and material properties of the components making up the system on the overall deflection of the entire sandwich structure and the analytical predictions showed good agreement with corresponding Finite Element simulations used to validate the results. The stiffness and thickness of the sandwich core are the main factors which determine the maximum deflection and resultant moment generated by the actuator upon activation. In addition, both methods indicate that the most efficient sandwich system design is one where the core is soft enough to act only as a means for joining the SMA skins with each other while at the same time, not providing a substantial stiffening effect which significantly reduces the axial actuation of the SMA-matrix skin layer. The results obtained from this work are expected to provide a major step towards the experimental design of SMAbased sandwich actuators and, potentially, the future implementation of these systems in applications requiring the generation of an actuated, reversible, specific bending deformation. In addition, it is hoped that this work will provide impetus for further research into the use and applications of SMA actuators using polymer composites as bias mechanisms.

\section{Acknowledgements}

This work was partially supported by MIUR with project Prin 2015 n.2015RT8Y45-PE8 on Smart Composite Laminates.

\section{Declaration of Conflict of Interest}

The authors declare no conflict of interest.

\section{References}

1. Lagoudas DC. Shape Memory Alloys. Vol. 1. Boston, MA: Springer US; 2008.

2. Spaggiari A, Castagnetti D, Golinelli N, Dragoni E, Scire Mammano G. Smart materials: Properties, design and mechatronic applications. Proc Inst Mech Eng Part L 
J Mater Des Appl. 2016;0(0):1-29.

3. Mohd Jani J, Leary M, Subic A, Gibson MA. A review of shape memory alloy research, applications and opportunities. Mater Des [Internet]. Elsevier Ltd; 2014;56:1078-113. Available from: http://dx.doi.org/10.1016/j.matdes.2013.11.084

4. Sun L, Huang WM, Ding Z, Zhao Y, Wang CC, Purnawali H, et al. Stimulusresponsive shape memory materials: A review. Mater Des [Internet]. Elsevier Ltd; 2012;33(1):577-640. Available from: http://dx.doi.org/10.1016/j.matdes.2011.04.065

5. Bodaghi M, Damanpack AR, Aghdam MM, Shakeri M. A phenomenological SMA model for combined axial-torsional proportional/non-proportional loading conditions. Mater Sci Eng A [Internet]. Elsevier; 2013;587:12-26. Available from: http://dx.doi.org/10.1016/j.msea.2013.08.037

6. Petrini L, Migliavacca F. Biomedical Applications of Shape Memory Alloys. J Metall [Internet]. 2011;2011(Figure 1):1-15. Available from: https://www.hindawi.com/archive/2011/501483/

7. Kuribayashi K, Tsuchiya K, You Z, Tomus D, Umemoto M, Ito T, et al. Selfdeployable origami stent grafts as a biomedical application of Ni-rich TiNi shape memory alloy foil. Mater Sci Eng A. 2006;419(1-2):131-7.

8. Biesiekierski A, Wang J, Gepreel MA, Wén C. A new look at biomedical Ti-based shape memory alloys. Acta Biomater [Internet]. Acta Materialia Inc.; 2012;8(5):16619. Available from: http://dx.doi.org/10.1016/j.actbio.2012.01.018

9. Yuan B, Zhu M, Chung CY. Biomedical Porous Shape Memory Alloys for HardTissue Replacement Materials. Materials (Basel). 2018;11:1716.

10. Kuo S, Shiau L, Chen K. Buckling analysis of shape memory alloy reinforced composite laminates. Compos Struct [Internet]. Elsevier Ltd; 2009;90(2):188-95. Available from: http://dx.doi.org/10.1016/j.compstruct.2009.03.007

11. Sun G. Bending of shape-memory alloy-reinforced composite beam. 1995;30:5750-4.

12. Wei ZG, Sandstrom R, Miyazaki S. Shape memory materials and hybrid composites for smart systems - Part II Shape-memory hybrid composites. J Mater Sci. 1998;33:3763-83. 
13. Cho HK, Rhee J. Nonlinear finite element analysis of shape memory alloy (SMA) wire reinforced hybrid laminate composite shells. Int J Non Linear Mech [Internet]. Elsevier; 2012;47(6):672-8. Available from: http://dx.doi.org/10.1016/j.ijnonlinmec.2011.11.002

14. Lu ZK, Weng GJ. A two-level micromechanical theory for a shape-memory alloy reinforced composite. Int J Plast. 2000;16:1289-307.

15. Ni DR, Ma ZY. Shape Memory Alloy-Reinforced Metal-Matrix Composites: A Review. Acta Metall Sin. 2014;27(5):739-61.

16. Birman V. Stability of functionally graded shape memory alloy sandwich panels. Smart Mater Struct. 1997;6:278-86.

17. Nejati M, Ghasemi-ghalebahman A, Soltanimaleki A, Dimitri R, Tornabene F. Thermal vibration analysis of SMA hybrid composite double curved sandwich panels. Compos Struct [Internet]. Elsevier; 2019;224(Mạrch):111035. Available from: https://doi.org/10.1016/j.compstruct.2019.111035

18. Ghaznavi A, Shariyat M. Non-linear layerwise dynamic response analysis of sandwich plates with soft auxetic cores and embedded SMA wires experiencing cyclic loadings. Compos Struct [Internet]. Elsevier Ltd; 2017;171:185-97. Available from: http://dx.doi.org/10.1016/j.compstruct.2017.03.012

19. Khalili SMR, Dehkordi MB, Carrera E, Shariyat M. Non-linear dynamic analysis of a sandwich beam with pseudoelastic SMA hybrid composite faces based on higher order finite element theory. Compos Struct [Internet]. Elsevier Ltd; 2013;96:243-55. Available from: http://dx.doi.org/10.1016/j.compstruct.2012.08.020

20. Dehkordi MB, Khalili SMR. Frequency analysis of sandwich plate with active SMA hybrid composite face-sheets and temperature dependent flexible core. Compos Struct [Internet]. Elsevier Ltd; 2015;123:408-19. Available from: http://dx.doi.org/10.1016/j.compstruct.2014.12.068

21. Samadpour M, Sadighi M, Shakeri M, Zamani HA. Vibration analysis of thermally buckled SMA hybrid composite sandwich plate. Compos Struct [Internet]. Elsevier Ltd;2015;119:251-63. Available from: http://dx.doi.org/10.1016/j.compstruct.2014.08.042 
22. Bodaghi M, Shakeri M, Aghdam MM. Thermo-mechanical behavior of shape adaptive composite plates with surface-bonded shape memory alloy ribbons. Compos Struct [Internet]. Elsevier Ltd; 2015;119:115-33. Available from:

http://dx.doi.org/10.1016/j.compstruct.2014.08.027

23. Bodaghi M, Damanpack AR, Aghdam MM, Shakeri M. Active Shape/Stress Control of Shape Memory Alloy Laminated Beams. Compos Part B Eng [Internet]. Elsevier Ltd; 2014;56:889-99. Available from:

http://dx.doi.org/10.1016/j.compositesb.2013.09.018

24. Damanpack AR, Bodaghi M, Aghdam MM, Shakeri M. Shape control of shape memory alloy composite beams in the post-buckling regime. Aerosp Sci Technol [Internet]. Elsevier Masson SAS; 2014;1:1-13. Available from: http://dx.doi.org/10.1016/j.ast.2014.06.004

25. Auricchio F, Petrini L. Improvements and algorithmical considerations on a recent three-dimensional model describing stress-induced solid phase transformations. Int $\mathbf{J}$ Numer Methods Eng. 2002;55(11):1255-84.

26. Auricchio F, Petrini L. A three-dimensional model describing stress-temperature induced solid phase transformations: Solution algorithm and boundary value problems. Int J Numer Methods Eng. 2004;61(6):807-36.

27. Souza AC, Mamiya EN, Zouain N. Three-dimensional model for solids undergoing stress-induced phase transformations. Eur J Mech A/Solids. 1998;17(5):789-806.

28. Mehrabi R, Kadkhodaei M. 3D phenomological constitutive modeling of shape memory alloys based on microplane theory. Smart Mater Struct. 2013;22(2):025017.

29. Lagoudas DC, Bo Z, Qidwai MA. A unified thermodynamic constitutive model for SMA and Finite Element Analysis of active metal matrix composites. Mech Compos Mater Struct. 1996;3(2):153-79.

30. Bodaghi M, Damanpack AR, Aghdam MM, Shakeri M. A robust three-dimensional phenomenological model for polycrystalline SMAs: Analytical closed-form solutions. Int J Eng Sci [Internet]. Elsevier Ltd; 2014;82:1-21. Available from: http://dx.doi.org/10.1016/j.ijengsci.2014.05.002

31. Brinson LC. One dimensional constitutive behaviour of shape memory alloys: 
thermomechanical derivation with non-constant material functions and redefined martensite internal variable. J Intell Mater Syst Struct. 1993;4:229-42.

32. Brinson LC, Lammering R. Finite element analysis of the behaviour of shape memory alloys and their applications. Int J Solids Struct. 1993;30:3261-80.

33. Ishii H, Ting KL. SMA actuated compliant bistable mechanisms. Mechatronics. 2004;14(4):421-37.

34. Nespoli A, Besseghini S, Pittaccio S, Villa E, Viscuso S. The high potential of shape memory alloys in developing miniature mechanical devices: A review on shape memory alloy mini-actuators. Sensors Actuators, A Phys. 2010;158(1):149-60.

35. Reynaerts D, Van Brussel H. Design aspects of shape memory actuators. Mechatronics. 1998;8(6):635-56.

36. Spaggiari A, Scirè Mammano G, Dragoni E. Optimum Mechanical Design of Binary Actuators Based on Shape Memory Alloys. Chapter "Smart Actuation Sens Syst Recent Adv Futur Challenges" Intech Publ [Internet]. 2012;1:3-34. Available from: http://www.intechopen.com/books/smart-actuation-and-sensing-systems-recentadvances-and-future-challenges/optimum-mechanical-design-of-binary-actuatorsbased-on-shape-memory-alloys

37. Ghosh P, Rao A, Srinivasa AR. Design of multi-state and smart-bias components using Shape Memory Alloy and Shape Memory Polymer composites. Mater Des [Internet]. Elsevier Ltd; 2013;44:164-71. Available from: http://dx.doi.org/10.1016/j.matdes.2012.05.063

38. Spaggiari A, Dragoni E. Analytical and numerical modeling of shape memory alloy Negator springs for constant-force, long-stroke actuators. J Intell Mater Syst Struct. 2014;25(9):1139-48.

39. Scirè Mammano G, Dragoni E. Increasing stroke and output force of linear shape memory actuators by elastic compensation. Mechatronics. 2011 Apr;21(3):570-80.

40. Simoneau C, Terriault P, Lacasse S, Brailovski V. Adaptive composite panel with embedded SMA actuators: Modeling and validation. Mech Based Des Struct Mach. 2014;42(2):174-92.

41. Pappada S, Gren P, Tatar K, Gustafson T, Rametta R, Rossini E, et al. Mechanical and 
Vibration Characteristics of Laminated Composite Plates Embedding Shape Memory Alloy Superelastic Wires. J Mater Eng Perform. 2009;18(5):531-7.

42. Bettini P, Riva M, Sala G, Di Landro L, Airoldi A, Cucco J. Carbon fiber reinforced smart laminates with embedded SMA actuators-part I: Embedding techniques and interface analysis. J Mater Eng Perform. 2009;18(5-6):664-71.

43. Riva M, Bettini P, Di Landro L, Sala G, Airoldi A. Carbon fiber-reinforced smart laminates with embedded SMA actuators-part II: Numerical models and empirical correlations. J Mater Eng Perform. 2009;18(5-6):672-8.

44. Turner TL, Buehrle RD, Cano RJ, Fleming GA. Design, fabrication, and testing of SMA enabled adaptive chevrons for jet noise reduction. SPIE Smart Struct Mater Smart Struct Integr Syst. 2004;5390(36):14-8.

45. Mizzi L, Spaggiari A, Dragoni E. Design-oriented modelling of composite actuators with embedded shape memory alloy. Compos Struct [Internet]. Elsevier; 2019;213(January):37-46. Available from: https://doi.org/10.1016/j.compstruct.2019.01.057

46. Sittner P, Hara Y, Tokuda M. Experimental study on the thermoelastic martensitic transformation in shape memory alloy polycrystal induced by combined external forces. Metall Mater Trans A. 1995;26(11):2923-35.

47. Timoschenko S, Goodier JN. Theory of Elasticity. Engineering Societies Monographs; 1951.

48. Hibbeler RC. Mechanics of Materials. 8th ed. 2001.

49. Nemat-Nasser S, Guo WG. Superelastic and cyclic response of NiTi SMA at various strain rates and temperatures. Mech Mater. 2006;38(5-6):463-74.

50. Liu Y, Xie Z, Van Humbeeck J. Cyclic deformation of NiTi shape memory alloys. Mater SciEng A. 1999;273-275:673-8.

51. Scirè Mammano G, Dragoni E. Functional fatigue of Ni-Ti shape memory wires under various loading conditions. Int J Fatigue. 2014;69:71-83. 